\title{
Recent progress of photocathodes for PMTs
}

\section{Motohiro Suyama ${ }^{1}$}

Electron Tube Division, Hamamatsu Photonics K.K.

314-5 Shimokanzo, Iwata 438-0193, Japan

E-mail: suyama@etd.hpk.co.jp

\section{Kimitsugu Nakamura}

Electron Tube Division, Hamamatsu Photonics K.K.

314-5 Shimokanzo, Iwata 438-0193, Japan

E-mail:kimitugu@etd.hpk.co.jp

The quantum efficiency (QE), the conversion efficiency of a photon to an electron, is one of the most important performance of photon sensors. In general, the QE of opto-semiconductor devices is superior to that of photocathodes for PMTs. As a result of our continuous efforts to improve them, an ultra bialkali photocathode (UBA) released a few years ago shows QE higher than $40 \%$ at $380 \mathrm{~nm}$, GaAsP does $50 \%$ in $550 \mathrm{~nm}$, and GaAs $30 \%$ in $750 \mathrm{~nm}$. In the case of UBA, however, linearity is a problem, when it is cooled down to liquid xenon temperature (-108 degree centigrade) for dark matter experiments. For these experiments, a high linearity photocathode at low temperature has been developed. This photocathode shows a little less QE than UBA, but much lower dark current than UBA even at room temperature, thus it's a good compromise in $\mathrm{S} / \mathrm{N}$ ratio. Including these topics, recent progresses of photocathode is described.

International Workshop on New Photon Detectors (PD09) Shinshu University Matsumoto Japan

24-26 June 2009

\footnotetext{
$1 \quad$ Speaker
} 


\section{Introduction}

The quantum efficiency (QE), the conversion efficiency of a photon to an electron, is one of the most important performance of photon sensors. Opto-semiconductor devices, such as silicon Photo-Diode (PD) utilizing photo-voltatic effect, have QE of higher than 70\% from ultraviolet to near infrared, as shown in Fig. 1.

Photoelectric effect used as a photocathode for a PMT, is a well known phenomenon occurs on most materials. However, the QE is very small, in general, the order of $10^{-4}$. The QE can be improved by three orders of magnitude to deposit a few alkali materials on a antimonide film to lower a work function. The photocathode produced like this is called an alkaliantimonide photocathode, where a bialkali photocathode consists of a potasium and a cesium, and a multialkali photocathode further includes a sodium. Even with alkali-antimonide photocathodes for PMTs, QE is less than 30\%, as shown in Fig. 1. Because of a high gain around $10^{6}$, fast response time of a few nano-seconds, a large effective area up to 20 inches with low dark current of $2 \times 10^{-20}\left(\mathrm{~A} / \mathrm{mm}^{2}\right)$, a PMT is a perfect photon sensor if QE is high. Therefore, Hamamatsu Photonics K.K. has been dedicating to improve it, and some of the results are reported here.

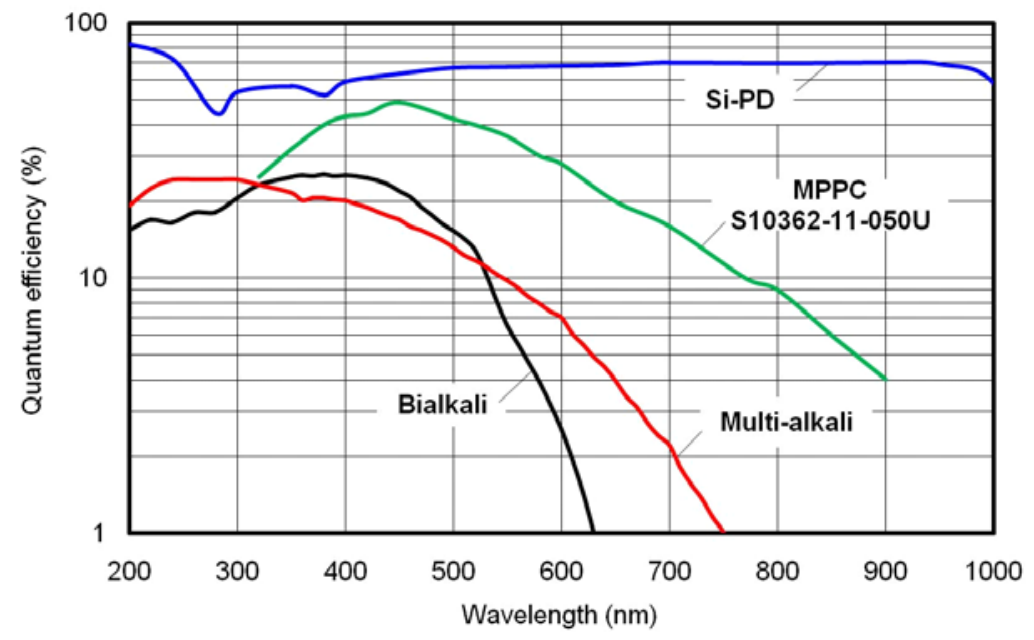

Figure 1: QE of several photon sensors. QE of a Si-PD utilizing photovoltatic effect is high about $70 \%$ from 200 to $1,000 \mathrm{~nm}$. QE of a bialkali or a multialkali photocathodes utilizing photoelectric effect is smaller than that of Si-PD.

\section{Ultra bialkali (UBA) photocathode}

A photocathode is considered as a semiconductor whose band diagram is shown in Fig. 2. Then, the photoelectric effect for photocathode can be explained by a three-step model [1].

Step1: Absorption of photons to generate electrons in the photocathode.

Step2: Diffusion of elctrons toward the boundary to vacuum.

Step3: Emission from the photocathode to vacuum. 
In order to improve the QE, we tried to improve crystallinity of a photocathode, because we believe crystallinity relates to every step in the three-step model. The QE of a bialkali photocathode is actually improved by approximately a factor of two as shown in Fig. 3, when the crystallinity of antimony film was improved [2]. We named this photocathode "ultra-bialkali (UBA)" and "super-bialkali (SBA)" photocathodes [3], and released in the market in 2007. As shown in Fig. 3, UBA shows QE of $43 \%$ at $380 \mathrm{~nm}$.

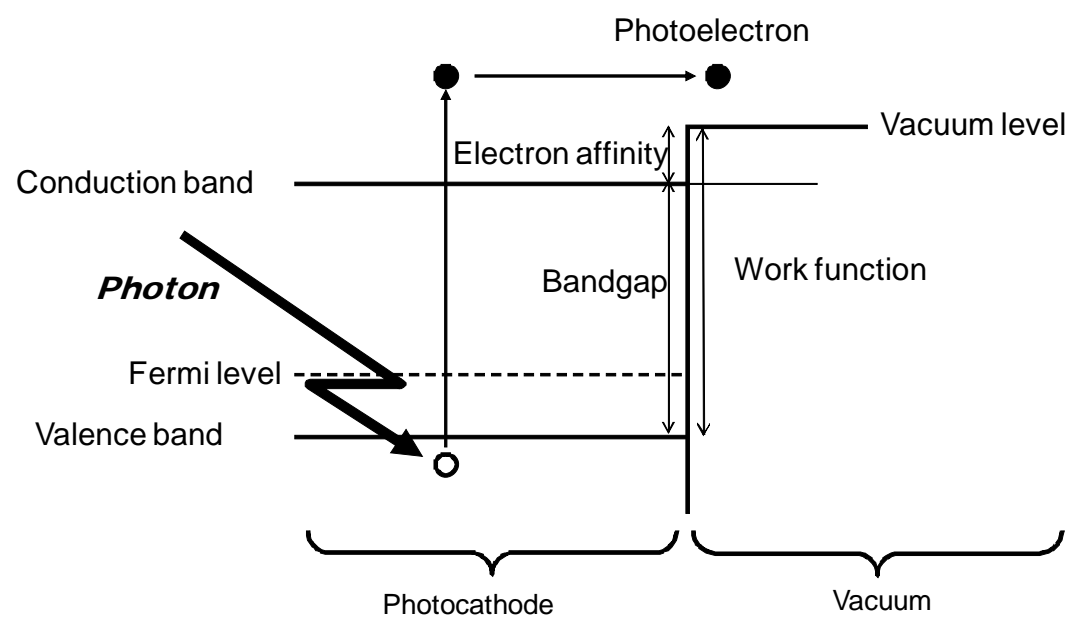

Figure 2: A band model of a photocathode. When a photon is absorbed, an electrons is excited to a conduction band (step 1), the electron diffuses to the boundary to vacuum (step 2). The electron emits to vacuum (step 3), if the electron has a larger energy than the work function.

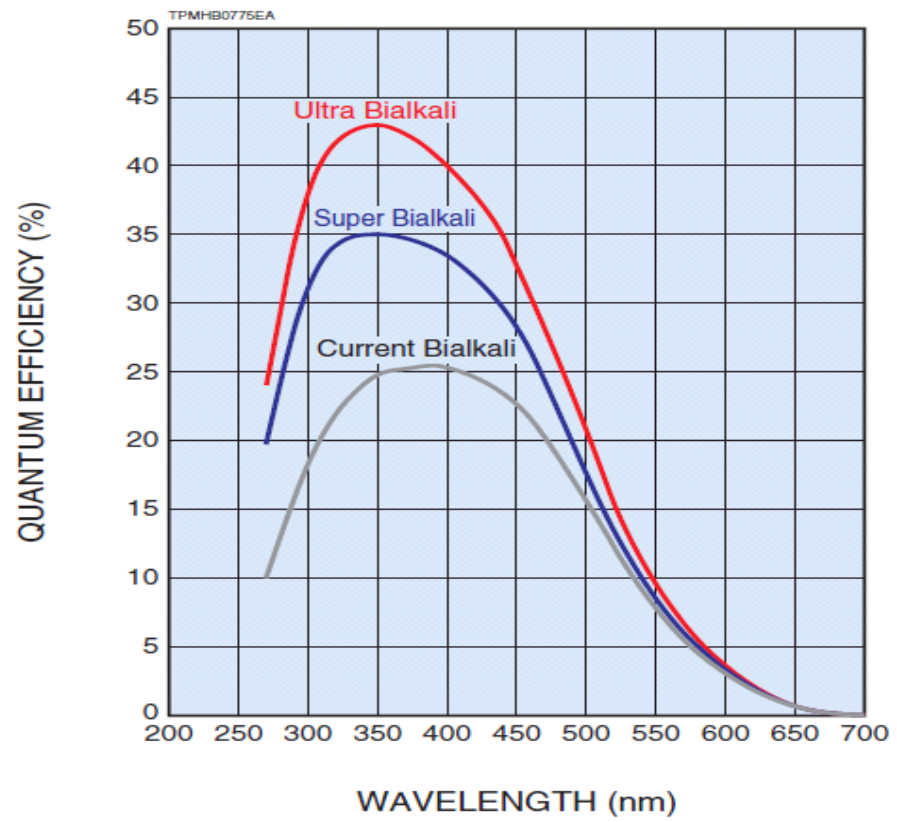

Figure 3: QE of ultra and super bialkali photocathodes. QE of ultra bialkali reaches $43 \%$ at $380 \mathrm{~nm}$, approximately a factor of two improvement from the conventional one. 


\section{New bialkali photocathode for low temperature}

UBA is still not perfect in terms of QE, and we keep trying to improve it further. In addition, we noticed that UBA shows poor linearity at low temperature like liquid Xe environment (-108 degree centigrade), due to high resistivity of photocathode at this temperature. The saturation current of conventional bialkali photocathode is shown in Fig. 4, as a function of temperature [4]. UBA shows similar characteristic, and considered not to be used for dark matter experiments at liquid Xe temperature.

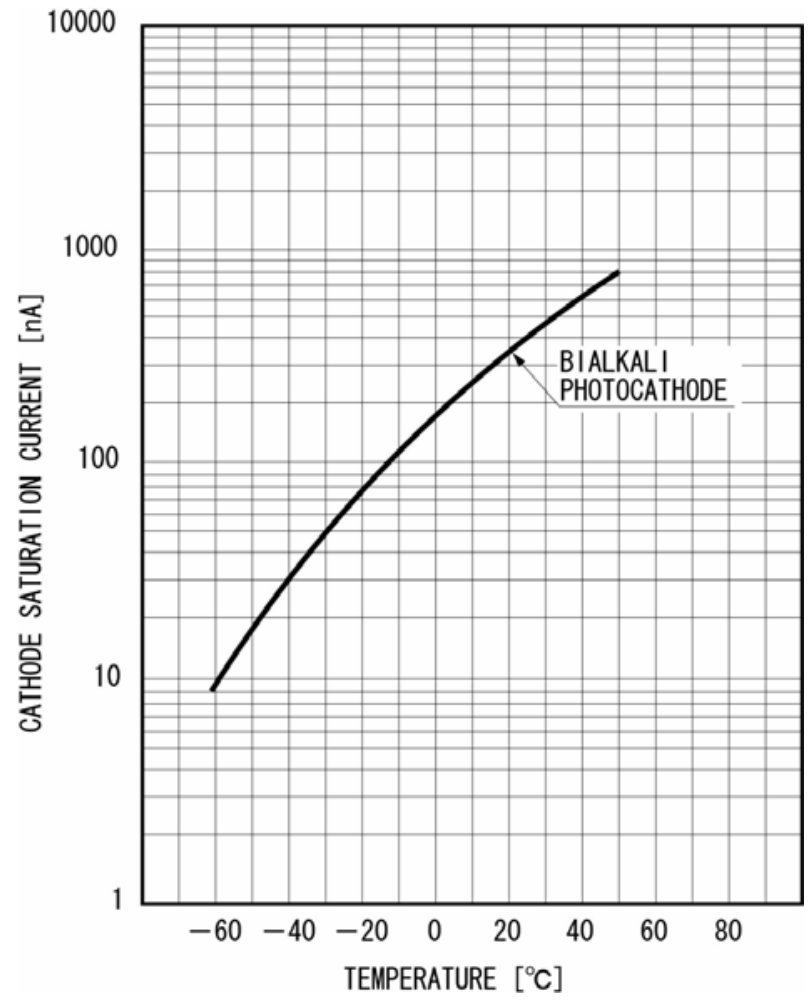

Figure 4: Saturation current of conventional bialkali photocathode.

In general, a radical aluminum pattern is fabricated under the photocathode to divide a large photocathode into several small ones to supply enough current even at low temperature. In this case, sufficient current is supplied at liquid Xe temperature, but not enough for liquid $\mathrm{Ar}$ temperature (-186 degree C). For liquid Ar temperatrure, a thin platinum underlay is used at the sacrifice of QE. As explained, there is no good photocathode for low temperature.

To solve this problem, we have developed a new bialkali photocathode with a little bit of bismuth to decrease the resistivity of photocathode [2]. As a result, the linearity of photocathode current is improved as shown in Fig. 5. The linearity is much higher than that of a conventional bialkali photocathode, and comparable to that of platinum underlay at -100 degree C. As shown in Fig. 6, QE of new photocathode is better than the others in almost all wavelength. At the scintillation wavelengh of Xe $(172 \mathrm{~nm})$, a conventional bialkali photocathode tuned to this wavelength is the best at this moment. $420 \mathrm{~nm}$ is another key wavelength for liquid $\mathrm{Ar}$ 
experiments, because scintilation light from $\mathrm{Ar}(130 \mathrm{~nm})$ is converted to this by a wavelength shifter.

As a by-product of a new bi-alkali photocathode, we confirmed that the dark current is approximately $1 / 3$ of UBA. It's worth to note that UBA shows a few times higher dark current than that of conventional bialkali photocathode. So the new photocathode shows lower dark current, while maintaining higher QE than conventional one, thus good compromize for signal to noise ratio at room temperature.
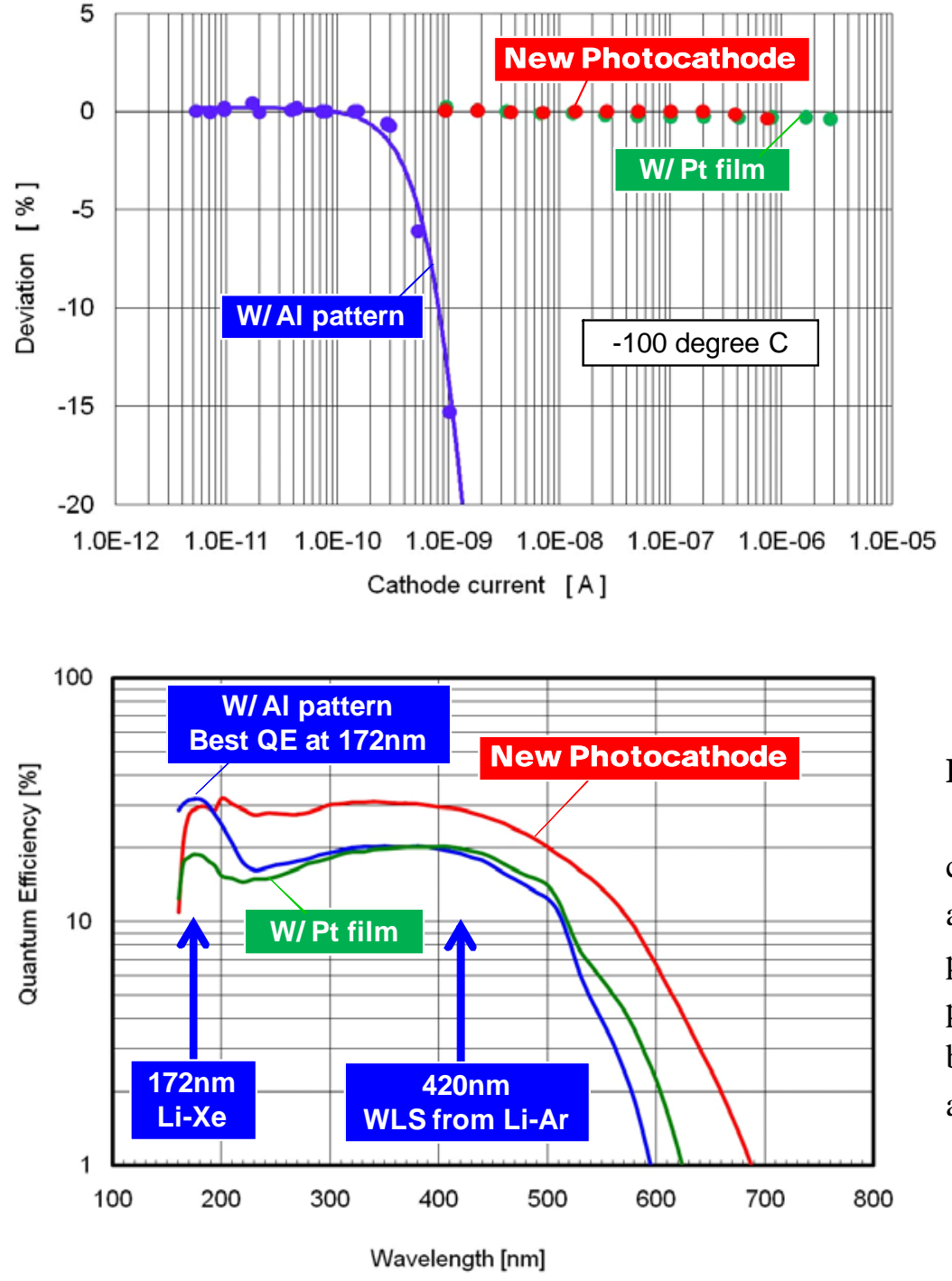

Figure $\quad 5$ : Linearity of new bialkali photocathode, comparing to the ones with aluminum electrode or platinum underlay. New photocathode shows much better linearity than that with aluminum pattern.

Figure 6: $Q E$ of new photocathode, comparing to ones with aluminum pattern or plutinum underlay. New photocathode shows better QE than the others at almost all wavelength.

\section{Potential of photocathode}

It is described above that the crystallity of photocathode affects QE, in terms of the absorption, the diffusion, and the emission. Much better crystallity is realized with semiconductor crystal, such as GaAs or GaAsP crystal. When properly activated by cecium, these material have negative electron affinity, thus high emission efficiency to vacuum can be 
achievable. As a matter of fact, GaAs crystal shows QE of 30\% from visible to near infrared region, as shown in Fig. 7. GaAsP crystal, which has a wider band gap thus stronger negative electron affinity than that of GaAs, shows QE of higher than 50\%. The spectral response of champion GaAsP photocathode shown in Fig. 7 achieves $63 \%$ at 500nm. This QE is the world record of photocathodes in visible region, as far as the authers knows, and shows the potential of photoelectric effect. In addition, Fig. 7 shows that QE of the photocathodes is close to that of Si-PD in some wavelength. Challenges to improve QE of photocathodes are under way.

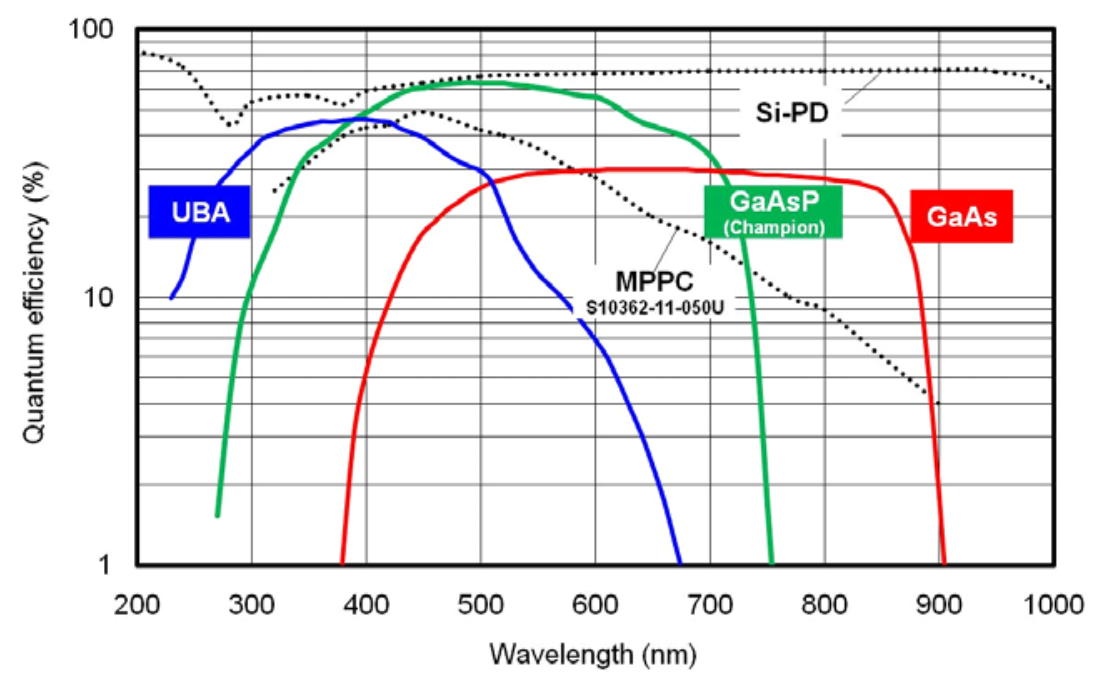

\section{Acknowledgements}

The authers thanks to Y. Hamana and Y. Ishigami for providing us data for new bialkali photocathodes. The authers also express our gratitude to T. Nagai and A. Kibune for fruitful discussion about semiconductor crystal photocathodes.

\section{References}

[1] A. H. Sommer, Photoemissive Materials, Robert E. Krieger Publishing Company, 1980

[2] K. Nakamura et al., Latest Bialkali Photocathode with Ultra High Sensitivity, Nucl. Instrum. Methods. , to be published.

[3] Hamamatsu Photonics K. K., UBA/SBA Photomultiplier Tube Series, 2008

[4] Hamamatsu Photonics K. K., Photomultiplier handbook (third edition), p. 235, 2007.
GaAs and champion data of GaAsP photocathodes, comparing to those of Si-PD, MPPC, and UBA. The champion data of GaAsP shows potential of photoelectric effect. 